\title{
Control of an Active Filter by Duty Cycle Modulation (DCM) for the Harmonic Decontamination of a Three-Phase Electrical Network
}

\author{
Théodore Patrice Nna Nna, Salomé Ndjakomo Essiane, Stève Pérabi Ngoffé \\ Technology and Applied Sciences Laboratory, University of Douala, Douala, Cameroon \\ Email:UFD-SCA@gmail.com, etknna@yahoo.fr
}

How to cite this paper: Nna, T.P.N., Essiane, S.N. and Ngoffé, S.P. (2020) Control of an Active Filter by Duty Cycle Modulation (DCM) for the Harmonic Decontamination of a Three-Phase Electrical Network. Journal of Power and Energy Engineering, 8, 1-14. https://doi.org/10.4236/jpee.2020.87001

Received: May 27, 2020

Accepted: July 27, 2020

Published: July 30, 2020

Copyright (อ 2020 by author(s) and Scientific Research Publishing Inc. This work is licensed under the Creative Commons Attribution International License (CC BY 4.0).

http://creativecommons.org/licenses/by/4.0/ (c) (i) Open Access

\begin{abstract}
This paper presents the control of the active filter by Duty Cycle Modulation (DCM) for the harmonic pollution control of a three-phase electrical system. Several research works have preceded this work, but most of them deal with the control by pulse-width modulation and the differences lie around the THD reduction performance, the quality of the wave obtained, the simplicity of the scheme and the cost of the control. For this purpose, we propose a very innovative approach to active filter control which aims at reducing the current THD at a lower rate than pulse-width modulation techniques improving at the same time the wave quality with a more simplified control scheme. First we present the schematic diagram of the active filter, then we present the design of the control of this active filter based on the real and imaginary instantaneous power method which uses the algebraic "Clark" transformation. The Duty Cycle Modulation controller is used to obtain the required control commands to be injected to the inverter. We then developed a simulink model of the active filter to validate this study. The simulations were performed in the Matlab/Simulink environment. The results obtained show a significant improvement on the harmonic current control techniques for the THD of $1.02 \%$ which was $26.25 \%$, the elimination of noise in the signal and the simplicity of the control with an easier implementation than the pulse width modulation (PWM) make the Duty Cycle Modulation (DCM) control a very promising tool.
\end{abstract}

\section{Keywords}

PWM, DCM, Active Filter, Inverter 


\section{Introduction}

The quality of power in power grids is the subject of much research. These networks include power lines operated at different voltage levels, connected in substations, and the managers must ensure the dynamic management of the whole production, transmission, consumption chain.

The incorporation of power electronic devices with non-linear characters in these electrical networks is partly responsible for the disturbances observed in the connection nodes of the networks which do not allow a better management of the network. A frequency analysis of the wave taken from the network reveals the presence of harmonic components and these are the cause of the malfunctions observed when the THD no longer complies with the standards.

There are several approaches to combat these disturbances, the first called load conditioning ensures equipment is less sensitive to energy disturbances. A second solution proposed by [1] consists in confining the harmonics for specific loads, by using special transformer couplings, and the third one consists in using filtering techniques:

Passive filters prevent harmonic currents from propagating in power networks and compensate for reactive power. However, they lack adaptability to changes in network and load impedance. The use of active filters avoids the disadvantages of passive filters. Three topologies are encountered in this respect, serial active filter (FAS), parallel active filter (FAP) and hybrid active filter (FAH). This during the parallel active filter (FAP) is the most used solution for the decontamination of harmonic currents. Several research works have been carried out around the control of the filter from its medium model [2] or the direct and hybrid control, the linear and non-linear control [3].

There are many strategies for controlling the parallel active filter (FAP) [4] but the differences lie in the THD reduction performances, the quality of the obtained wave, the simplicity of the scheme and the cost. The latter will be used in our work.

In the control of active filters, pulse width modulators (PWMs) are the most widely used and have proven performance due to their immunity to noise. However, they are very complex to implement and filtering when recovering the transmitted signal is not easy.

A new duty-cycle modulator (DCM) has been developed by [5] and is based on the non-linear operation of an operational amplifier acting as a voltage-controlled oscillator in high frequencies. Its simplicity of implementation, simple mathematical foundations and spectral poverty are real assets. Applications in the field of power electronics device control are still under development [6] [7] and the results reflect these potentialities.

The objective of this article is to control a parallel active filter by Duty Cycle Modulator while improving the quality of electrical power. To validate our results, we compare two active filter control strategies: pulse width modulation (PWM) and duty-cycle modulator (DCM). 
To carry out this work, we first present the active filter and its principle scheme, then we present a methodological approach to the control of the active filter by Duty Cycle Modulation. The design of the control of this active filter is based on the method of real and imaginary instantaneous powers and the DCM controller is used to obtain the required control commands to be injected to the inverter. We will then develop a simulink model of the active filter to validate this study and present our results and a brief discussion will be made. The simulations will be performed in the Matlab/Simulink environment.

\section{Active Filter}

The inverter is the essential part of the active filter. Depending on the type of coupling filter that connects it to the grid and the passive element that serves as its energy source, it will be a voltage or current inverter for this purpose. The inverter can be voltage or current controlled and can therefore act as a voltage or current source with respect to the external circuit. In practice, depending on the type of correction to be made to the grid, one structure is preferred to the other if it makes it easier and cheaper to build the inverter-control assembly.

Several configurations have been presented in the literature, the most studied being:

- Parallel active filter with voltage structure.

- Parallel active filter with current structure.

\section{Active Parallel Filter and Operating Principle}

Figure 1 shows the synoptic diagram and the operating principle of a parallel active filter.

In the configuration of Figure 1, the load absorbs the harmonically disturbed currents $i_{l i}(t)$ which the filter must compensate by injecting the currents $i_{f i}(t)$, so that the source only provides the active currents $i_{s i}(t)$. We write for the load:

$$
i_{l i}(t)=i_{l i, a}(t)+i_{l i, r h}(t)
$$

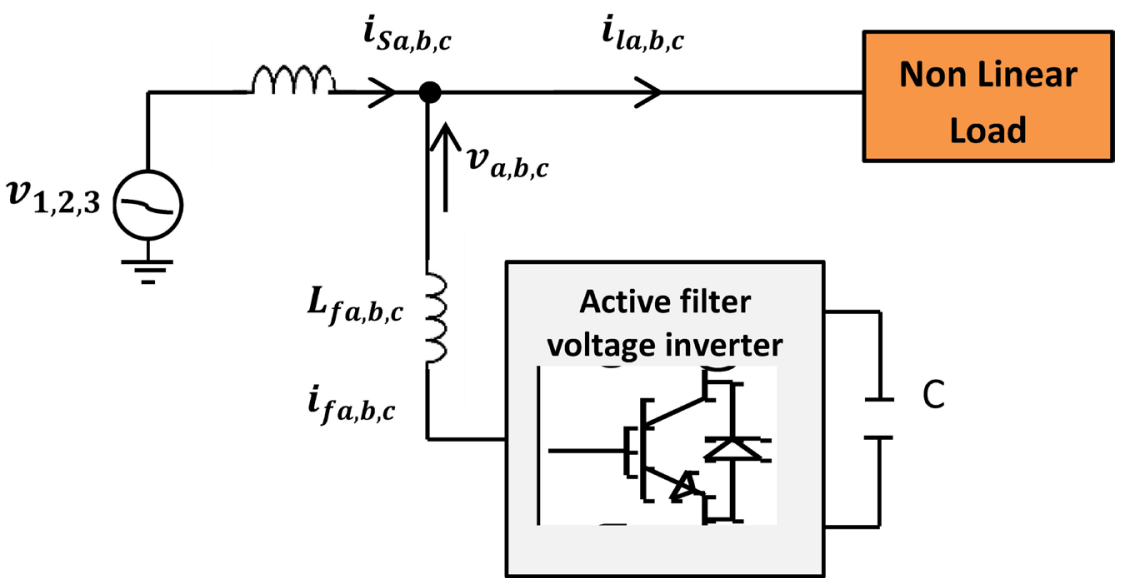

Figure 1. Schematic diagram of the parallel active filter. 
With, $i=a, b, c, i_{l i, a}(t)$ is the active part of the load current, $i_{l i, r h}(t)$ is the reactive and harmonic part of the load current.

And for the source, considering that the desired currents are active, $i_{s i, a}(t)$, and correspond mostly to the active part necessary for the load $i_{l i, a}(t)$ next to a small current $i_{\text {pertes }, i}(t)$ which will be absorbed by the filter to compensate for these losses. We will have:

$$
i_{s i}(t)=i_{s i, a}(t)+i_{\text {pertes }, i}(t)
$$

The law of nodes at Pcc in Figure 1 implies:

$$
i_{s i}(t)+i_{f i}(t)=i_{l i}(t)
$$

Replacing (1) and (2) in (3) gives:

$$
i_{s i, a}(t)+i_{\text {pertes }, i}(t)+i_{f i}(t)=i_{l i, a}(t)+i_{l i, r h}(t)
$$

However, the active current supplied by the source $i_{s i, a}(t)$ corresponds to the active part $i_{l i, a}(t)$ requested by the load,

$$
i_{f i}(t)=i_{l i, r h}(t)-i_{\text {pertes }, i}(t)
$$

Equation (5) summarizes the principle of compensation expected by the active filter.

\section{Methodological Approach to Active Filter Control by Duty Cycle Modulation}

We propose an architecture in which the control strategy of the duty-cycle modulator (DCM) voltage inverter is based on the identification and determination of reference currents using the method of real and imaginary instantaneous powers using the algebraic "Clark" transformation. These reference currents are then injected into the duty-cycle modulator which generates the control commands for the inverter, then demodulated by an LC filter and injected into the grid in phase opposition. The PI regulator is applied to regulate the DC bus voltage.

\subsection{Identification and Calculation of Harmonic Currents}

The current absorbed by the pollutant load is composed of a fundamental and harmonics. The active filter is used to generate harmonic currents of the same amplitude but in phase opposition to those existing in the load. To do this, the harmonic currents of the load must be identified. Several identification methods exist [3] [4]:

- The first is based on the spectral analysis of the pollutant current.

- The second uses a bandpass filter to filter the fundamental.

- The third uses the notions of instantaneous real and imaginary powers.

The latter is the most widely used in most active filters because it achieves the best match between static and dynamic performance.

This method uses the "Clark" algebraic transformation to transform the three-phase current and voltage systems presented in the $a, b, c$ benchmark to a 
new benchmark $\alpha, \beta$ as follows:

Voltage components:

$$
\left[\begin{array}{l}
V_{\alpha} \\
V_{\beta}
\end{array}\right]=\sqrt{\frac{2}{3}}\left[\begin{array}{ccc}
1 & -\frac{1}{2} & -\frac{1}{2} \\
0 & \frac{\sqrt{3}}{2} & -\frac{\sqrt{3}}{2}
\end{array}\right]\left[\begin{array}{l}
V_{a} \\
V_{b} \\
V_{c}
\end{array}\right]
$$

Current components:

$$
\left[\begin{array}{c}
I_{\alpha} \\
I_{\beta}
\end{array}\right]=\sqrt{\frac{2}{3}}\left[\begin{array}{ccc}
1 & -\frac{1}{2} & -\frac{1}{2} \\
0 & \frac{\sqrt{3}}{2} & -\frac{\sqrt{3}}{2}
\end{array}\right]\left[\begin{array}{l}
I_{a} \\
I_{b} \\
I_{c}
\end{array}\right]
$$

In a marker $a, b, c$, the instantaneous active power is given by:

$$
P_{3}(t)=V_{a} I_{a}+V_{b} I_{b}+V_{c} I_{c}
$$

Similarly, in the $\operatorname{tag} \alpha, \beta$ :

$$
P_{\alpha \beta}(t)=V_{\alpha} I_{\alpha}+V_{\beta} I_{\beta}
$$

where,

The actual instantaneous power:

$$
P=V_{\alpha} I_{\alpha}+V_{\beta} I_{\beta}=P_{\alpha}+P_{\beta}
$$

One of the advantages resulting from this transformation is the separation of homopolar current or voltage sequences.

[8] proposes the following definition of the instantaneous imaginary power:

$$
q=V_{\alpha} I_{\beta}-I_{\alpha} V_{\beta}
$$

This power can be written in the $a, b, c$ marker as follows:

$$
q(t)=-\frac{1}{\sqrt{3}}\left[\left(V_{a}-V_{b}\right) \cdot I_{c}+\left(V_{b}-V_{c}\right) \cdot I_{a}+\left(V_{c}-V_{a}\right) \cdot I_{b}\right]
$$

This expression is well known for the measurement of conventional reactive power in the three-phase system.

From relations (10) and (11), we can establish the following matrix relation:

$$
\left[\begin{array}{l}
P \\
q
\end{array}\right]=\left[\begin{array}{cc}
V_{\alpha} & V_{\beta} \\
-V_{\beta} & V_{\alpha}
\end{array}\right]\left[\begin{array}{c}
I_{\alpha} \\
I_{\beta}
\end{array}\right]
$$

The instantaneous real and imaginary powers, respectively $\mathrm{p}$ and $\mathrm{q}$, are given by the generalized terms:

$$
\begin{aligned}
& P=\bar{P}+\tilde{P} \\
& q=\bar{q}+\tilde{q}
\end{aligned}
$$

$\bar{P}$ is the DC part related to the fundamental active component of the current and $\bar{q}$ is the DC part related to the fundamental reactive component of the current. 
What we are interested in is to extract the components $\tilde{P}, \tilde{q}$, fluctuating parts related to the sum of the disturbance components of current and voltage. To achieve this, we apply the second order Butterworth filter.

To calculate the compensation currents of the reference in the coordinates $\alpha$, $\beta$, the expression (13) is inverted and becomes:

$$
\left[\begin{array}{c}
I_{\alpha}^{*} \\
I_{\beta}^{*}
\end{array}\right]=\frac{1}{V_{\alpha}^{2}+V_{\beta}^{2}}\left[\begin{array}{cc}
V_{\alpha} & -V_{\beta} \\
V_{\beta} & V_{\alpha}
\end{array}\right]\left[\begin{array}{l}
P \\
q
\end{array}\right]
$$

Thanks to the Concordia Inverse Transformation (TCI), the alternating parts of the $\tilde{P}$ and $\tilde{q}$, power ratings reduce three-phase disturbance currents. These currents represent the disturbances and become the reference currents that are to be injected in phase opposition into the power grid to eliminate harmonics. Relationship (7) therefore gives:

$$
\left[\begin{array}{c}
I_{a}^{*} \\
I_{b}^{*} \\
I_{c}^{*}
\end{array}\right]=\sqrt{\frac{2}{3}}\left[\begin{array}{cc}
1 & 0 \\
-\frac{1}{2} & \frac{\sqrt{3}}{2} \\
-\frac{1}{2} & -\frac{\sqrt{3}}{2}
\end{array}\right]\left[\begin{array}{c}
I_{\alpha}^{*} \\
I_{\beta}^{*}
\end{array}\right]
$$

The principle of Determination of the reference currents of the active filter by the classical method of the instantaneous active and reactive powers is described in Figure 2.

\subsection{Voltage Regulation}

Figure 3 shows the block diagram of $V_{d c}$ control with the proportional controller.

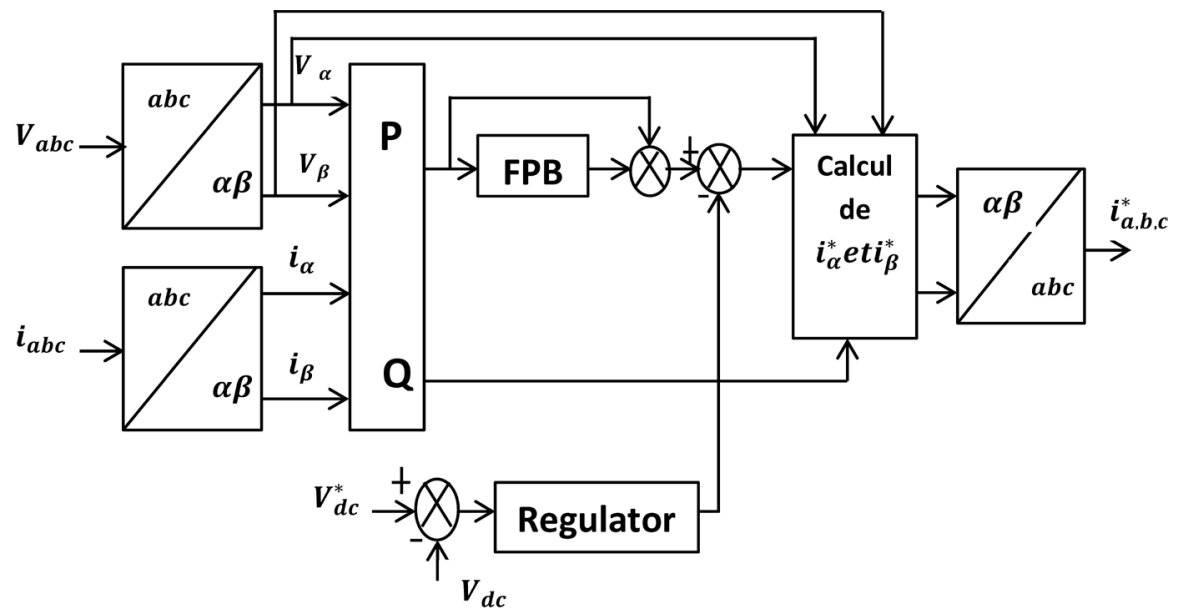

Figure 2. Reference current extraction algorithm.

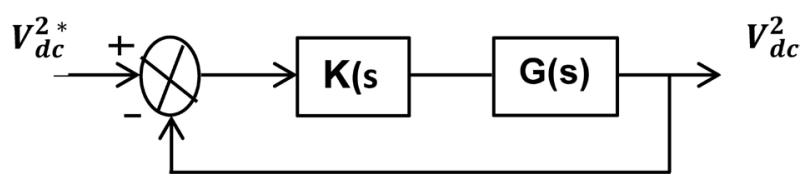

Figure 3. Block diagram of Vdc control with proportional controller. 
We have chosen the following PI regulator to regulate the capacitor voltage $V_{d c}$ to its set value $V_{d c}^{*} \quad[1]$ :

$$
K(s)=\frac{K_{r}}{1+\tau_{c}(s)}
$$

With: $K_{r}$ : Regulator gain; $\tau_{c}$ : Time constant.

The term $G(s)$ stands for:

$$
G(s)=\frac{2}{C(s)}
$$

After calculation, the closed-loop transfer function is of the form:

$$
F(s)=\frac{\omega^{2}}{S^{2}+2 \varepsilon_{c} \omega S+\omega^{2}}
$$

With: $\omega=\sqrt{\frac{2 K_{c}}{C \tau_{c}}}$ et $\varepsilon_{c}=\frac{1}{2 \sqrt{2}} \sqrt{\frac{C}{K_{c} \cdot \tau_{c}}}$

To achieve a good compromise between dynamic and static performance, we will choose a value of $\xi_{c}$ between 0.4 and 0.8 i.e. 0.7 .

\subsection{Optimal Duty-Cycle Modulation Current Control}

Duty Cycle Modulation (DCM) is a modulation technique that consists of varying the duty cycle as a function of the signal to be modulated. As the duty cycle is a non-linear function for the control of the signal, it is sufficient to develop an excellent linear approximation of the signal during the digital analysis by correctly setting the parameters of the circuit. In low frequency the Duty-Cycle Modulation controls the signal to be modulated. It is built around an operational amplifier operating in switching mode, i.e. it delivers an on/off signal at the output.

For this paper, we use the non-inverting Duty-Cycle Modulation (DCM).The principle of Duty Cycle Modulation Control is described in Figure 4.

The input signal $I_{r e f}=U_{m}$ is transformed into a periodic switching waveform.

We're posing $U_{m}=I_{\text {ref }}$
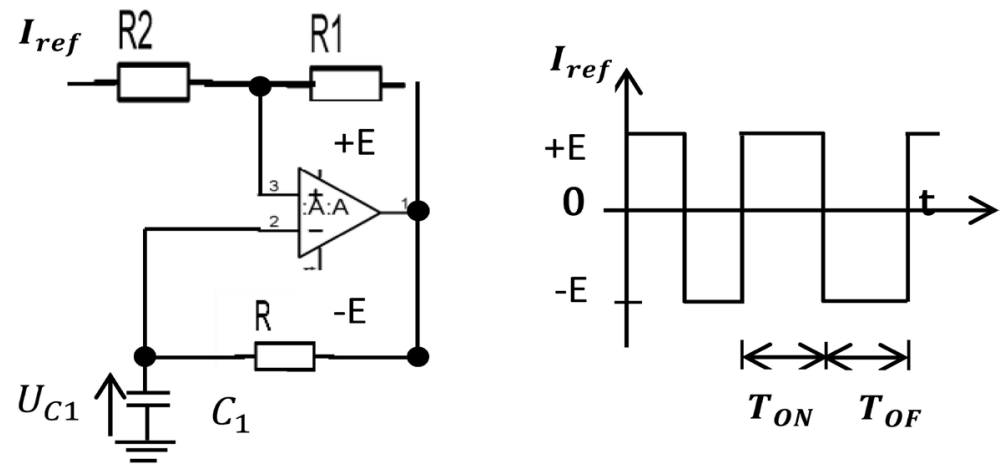

Figure 4. Current control by Duty-Cycle Modulation (DCM). 


$$
\begin{gathered}
u^{+}=\frac{\frac{U_{m}}{R_{1}}+\frac{U_{s}(t)}{R_{2}}}{\frac{1}{R_{1}}+\frac{1}{R_{2}}} \\
\Rightarrow u^{+}=\frac{U_{m} R_{2}}{R_{1}+R_{2}}+\frac{U_{s}(t) R_{1}}{R_{1}+R_{2}}
\end{gathered}
$$

With:

$$
\begin{gathered}
\alpha=\frac{R_{1}}{R_{1}+R_{2}} \\
1-\alpha=\frac{R_{2}}{R_{1}+R_{2}}=\alpha_{1}
\end{gathered}
$$

The AOP operating in switching mode we have:

$$
\begin{gathered}
U_{s}(t)= \begin{cases}E & \text { si } u^{+}>u^{-} \\
-E & \text { si } u^{+}<u^{-}\end{cases} \\
\Rightarrow u^{+}= \begin{cases}\alpha E+\alpha_{1} U_{m} & \text { si } U_{s}(t)=E \\
-\alpha E+\alpha_{1} U_{m} & \text { si } U_{s}(t)=-E\end{cases}
\end{gathered}
$$

The capacitor voltage variation is defined by:

$$
R C \frac{\mathrm{d} U_{c}(t)}{\mathrm{d} t}+U_{c}(t)=U_{s}(t)
$$

Where

$$
U_{c}(t)=\left(u_{i}-u_{f}\right) \mathrm{e}^{-\frac{t}{\tau}}+u_{f}
$$

With $\tau=R C$

The charging and discharging time of the capacitor are included respectively in: $0<t<T_{1}$ et $T_{1}<t<T_{2}$.

$$
T_{1}=-\tau \ln \frac{\alpha_{1} U_{m}+(\alpha-1) E}{\alpha_{1} U_{m}-(1-\alpha) E}
$$

Et

$$
\begin{gathered}
T_{2}=-\tau \ln \frac{\alpha_{1} U_{m}-(\alpha-1) E}{\alpha_{1} U_{m}+(1+\alpha) E} \\
T=\tau \ln \frac{\alpha_{1} u_{m}-(1+\alpha) E}{\alpha_{1} u_{m}+(\alpha-1) E}+\tau \ln \frac{\alpha_{1} u_{m}+(1+\alpha) E}{\alpha_{1} u_{m}-(\alpha-1) E} \\
\Rightarrow T=\tau \ln \left(\frac{\left(\alpha_{1} U_{m}\right)^{2}-((1+\alpha) E)^{2}}{\left(\alpha_{1} U_{m}\right)^{2}-((\alpha-1) E)^{2}}\right)
\end{gathered}
$$

The exact compensation of the load current requires above all a good precision on the extraction of this current. However, the control strategy of a FAP, shown in Figure 5, is based on 3 elementary blocks:

BLOCK 1, which is elementary for any control strategy. It is at this stage that 


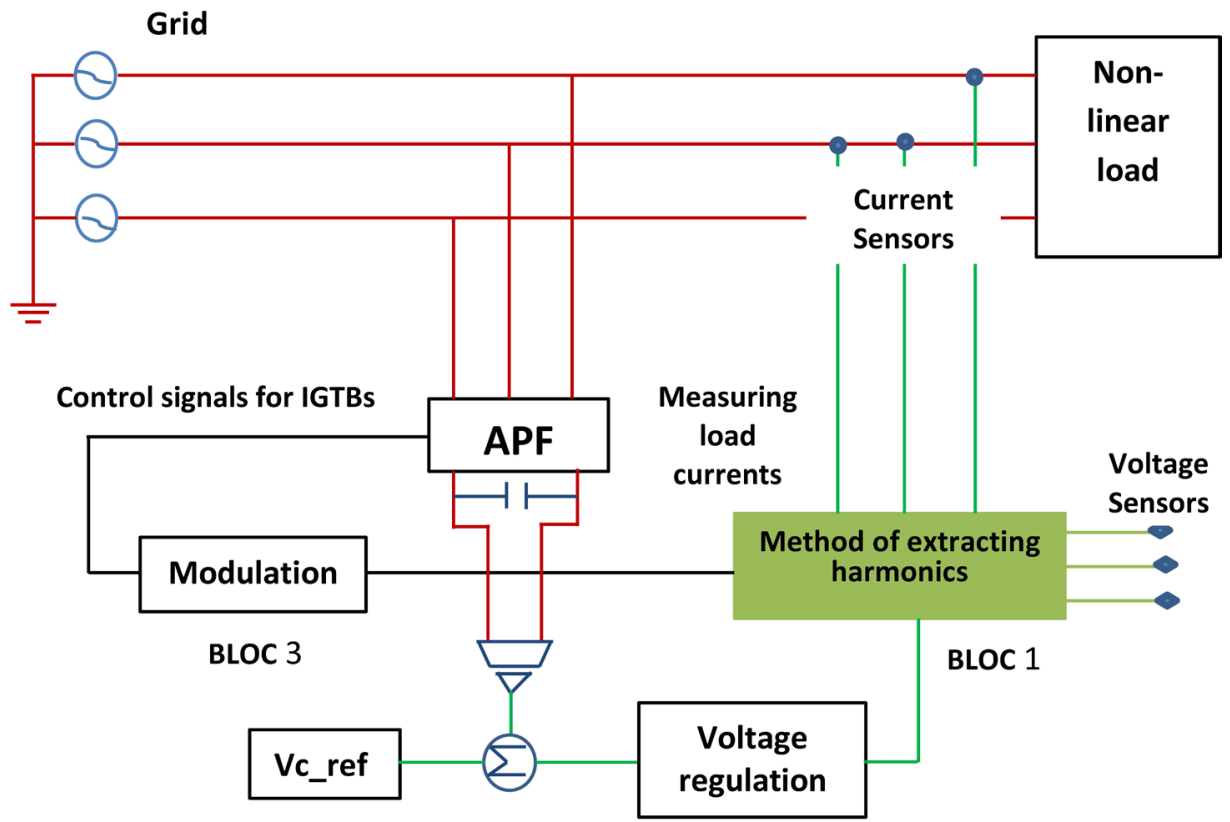

BLOC 2

Figure 5. FAP MRC control strategy.

the reference currents are determined;

BLOCK 2, allows to regulate the DC bus voltages and to determine, afterwards, the losses in the filter that will be absorbed from the network;

BLOCK 3, generates the PWM signals necessary to control the IGBTs from the reference signals already established and regulates the filter currents to their reference currents.

The duty cycle $R_{m}(u)$

$$
R_{m}\left(u_{m}\right)=\frac{T_{1}\left(U_{m}\right)}{T\left(U_{m}\right)} \Rightarrow R_{m}=\frac{\ln \frac{\alpha_{1} U_{m}-(1+\alpha) E}{\alpha_{1} U_{m}+(\alpha-1) E}}{\ln \left[\frac{\left(\alpha_{1} U_{m}\right)^{2}-((1+\alpha) E)^{2}}{\left(\alpha_{1} U_{m}\right)^{2}-((\alpha-1) E)^{2}}\right]}
$$

Modulator (DCM) whose optimal parameters are:

$$
\alpha=0.003081723734398 \text { ou } \alpha=\frac{R_{1}}{R_{1}+R_{2}}
$$

We use an Optimal Duty-Cycle

$$
\tau=0.000486670905896 \mathrm{~s} \text { ou } \tau=R C
$$

The base frequency of our MRC modulator has been set at 166,690 Hz. Then the AOP must be able to switch from 166,690 Hz. Then the AOP must be able to switch from $+V_{\text {sat }}$ à $-V_{\text {sat }}$ et de $-V_{\text {sat }}$ à $+V_{\text {sat }}$.

With $f_{\text {com }}>166690 \mathrm{~Hz}$ which is the switching frequency.

Figure 6 is the simulink model of the duty-cycle modulated current counter, which provides the control commands for the inverter's IGBT switches. 


\section{Results and Discussions}

The MATLAB/SIMULINK software is used, the simulated electrical network is 3-phase balanced 3-wire $100 \mathrm{~V}, 50 \mathrm{~Hz}$.

The pollutant load is represented by a conventional rectifier consisting of two thyristor bridges connected in series in an electrical network.

Figure 7 shows the block diagram of the circuit simulation, it consists of the three-phase mains voltage source, the non-linear load and the active filter.

The power circuit of a parallel active filter, based on an IGBT two-stage voltage inverter, placed between a three-phase grid and a non-linear graetz-bridge polluting load, is shown in Figure 8.

Simulation studies are performed for the system parameters represented in Table $1 a=0.003081723734398 ; a_{1}=0.996918277 ; \frac{1}{R C}=20547766$ or $\tau=R C$.

The graphs of the source current before application of the parallel active filter are given in Figure 9. The current is distorted with respect to the half-period point, which means that harmonics multiple of 2 and 3 are non-existent in the spectrum of the $i_{s a}$ current and only two ranks $(6 \mathrm{~h} \pm 1)$ are present; this is confirmed by the spectrum of $i_{s a}$ in Figure 10 .

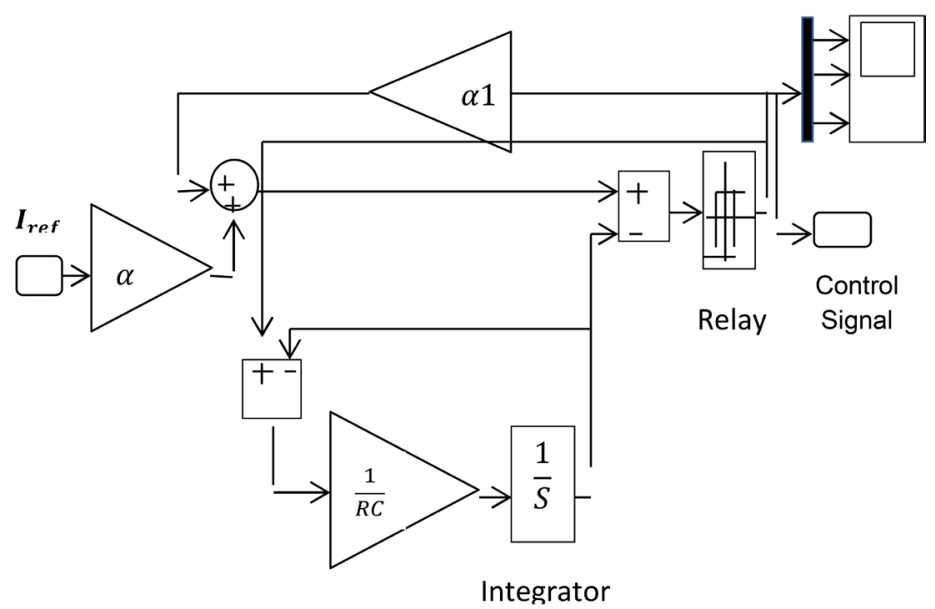

Figure 6. Duty-Cycle Modulation simulation scheme under Simulink.

GRID

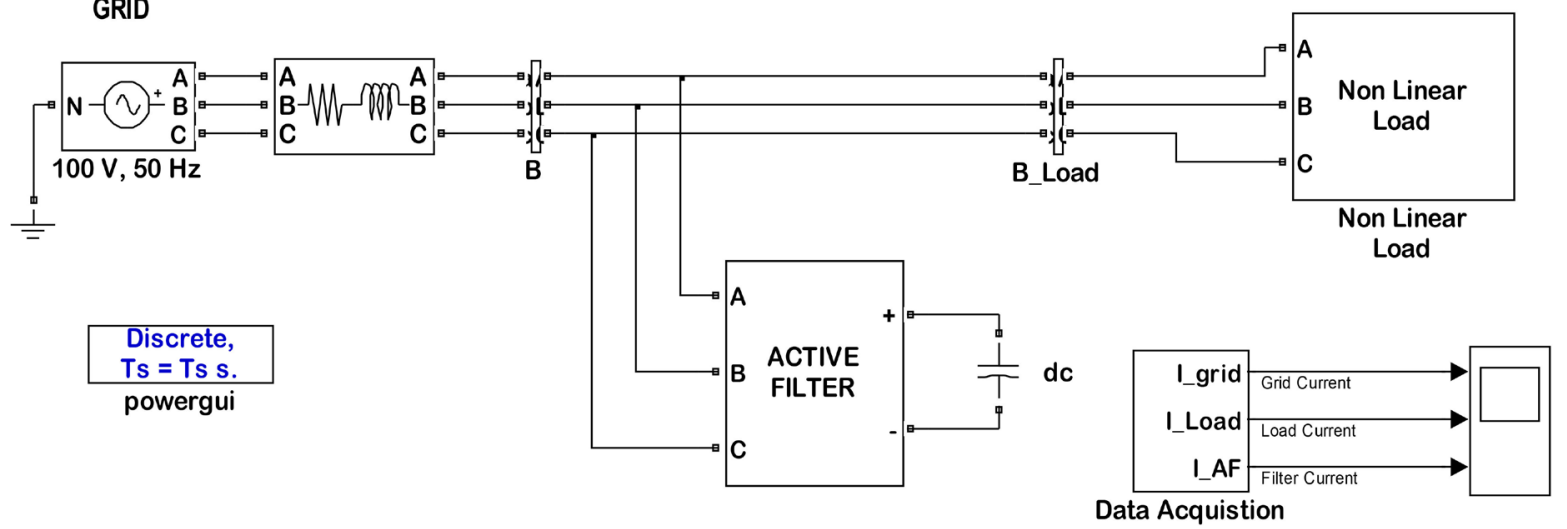

Figure 7. Block diagram of the circuit simulation. 


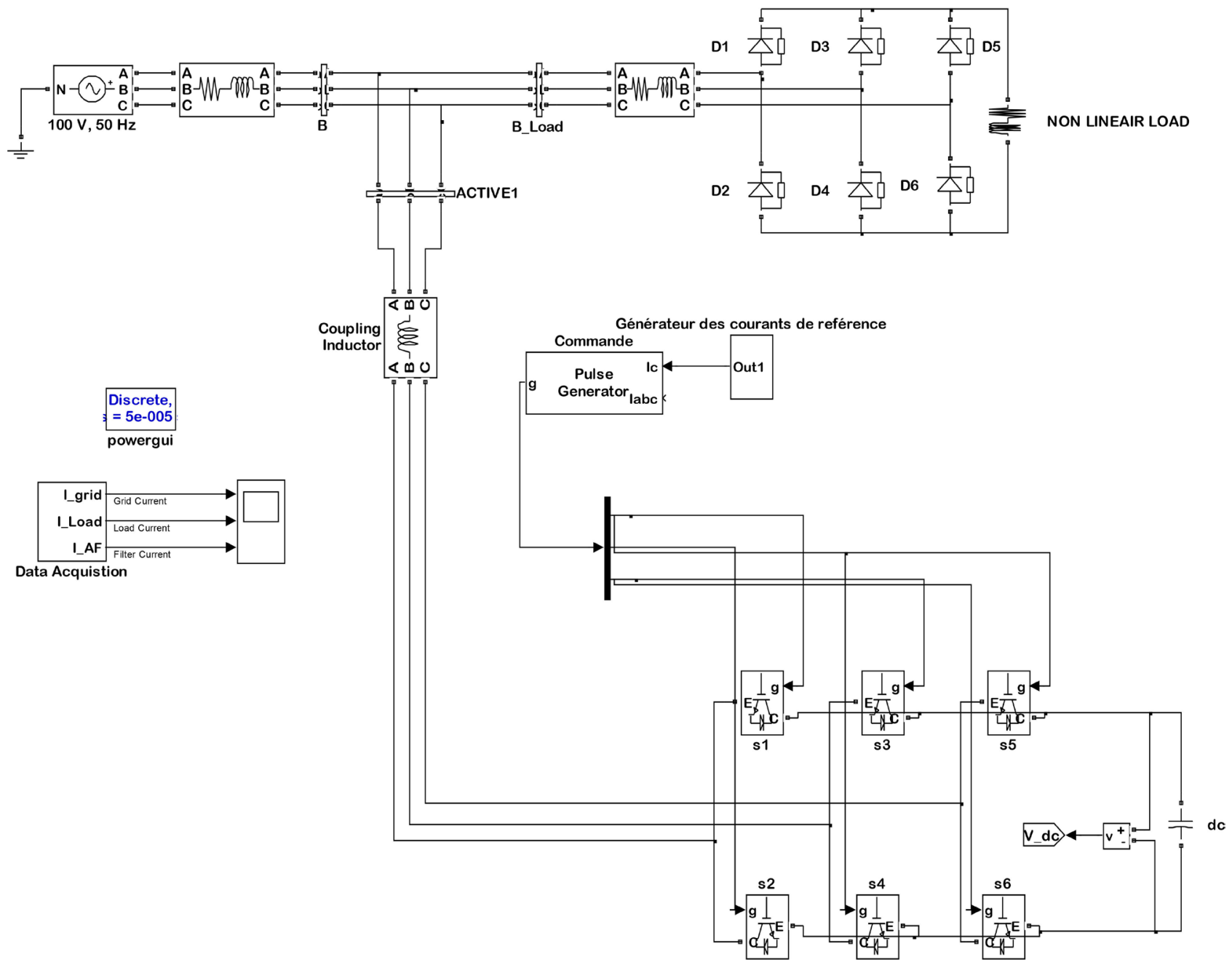

Figure 8. Circuit de puissance du filtre actif parallèle.

Table 1. Simulation parameters.

\begin{tabular}{cc}
\hline Settings & Numerical values \\
\hline RMS voltage & $100 \mathrm{~V}$ \\
Frequency & $50 \mathrm{~Hz}$ \\
Line resistance & $0.1 \Omega$ \\
Line inductance & $0.15 \mathrm{e}-3 \mathrm{H}$ \\
Load resistance & $60 \Omega$ \\
Load inductance & $20 \mathrm{e}-3 \mathrm{H}$ \\
Filter inductance Lf & $4 \mathrm{e}-3 \mathrm{H}$ \\
Cf & $15 \mu \mathrm{f}$ \\
Vdc & $440 \mathrm{~V}$
\end{tabular}

The spectral analysis of the grid current presented in (Figure 9) shows the presence of several ranks of harmonics disturbing the shape of the current waveform, i.e. a THD of $26.25 \%$. 
To validate our results, we apply to our active filter the pulse-width modulation control and we obtain after filtering the signal in Figure 11 which shows an improvement of the curve and whose spectrum in Figure 12 shows a clear decrease of the harmonics. Let THD $=1.78 \%$.

We then apply the Duty Cycle Modulation control to our active filter and after filtering we obtain the signal in Figure 13 which shows a significant improvement in the curve and whose spectrum in Figure 14 shows a greater reduction in harmonics. Let THD $=1.02 \%$.

According to the simulation results obtained in Figure 12 and Figure 14, the harmonic distortion rate (THD) of the current at the connection point (calculated on the first 20 harmonic ranks) is: $\mathrm{THD}=1.78 \%$ for pulse width control and $\mathrm{THD}=1.02 \%$ for duty cycle control, which corresponds to the IEEE STD

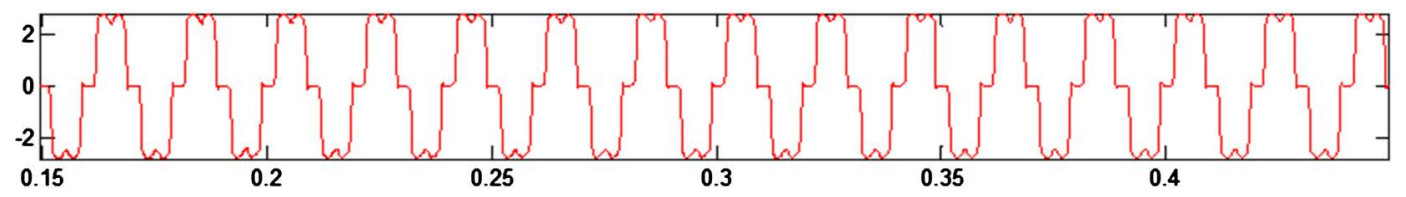

Figure 9. Current wave $i_{s a}$ before filtering.

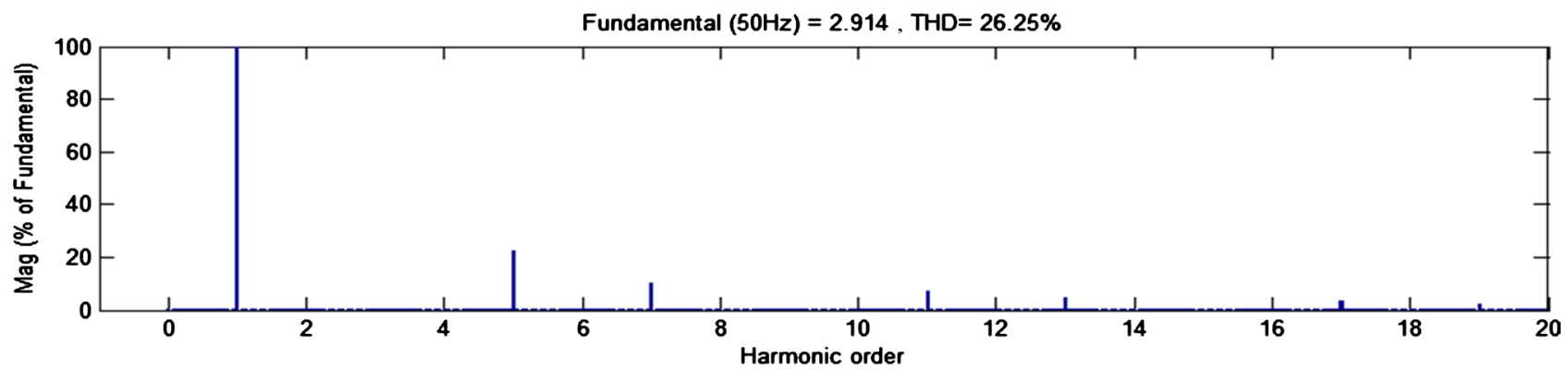

Figure 10. Current spectrum in the network before filtering.

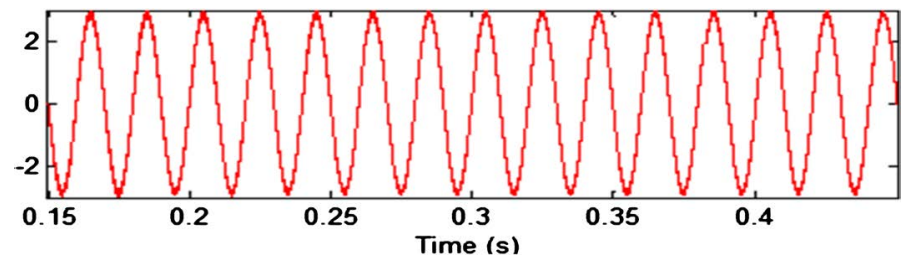

Figure 11. Current wave $i_{s a}$ after filtering for pulse width modulation PWM control.

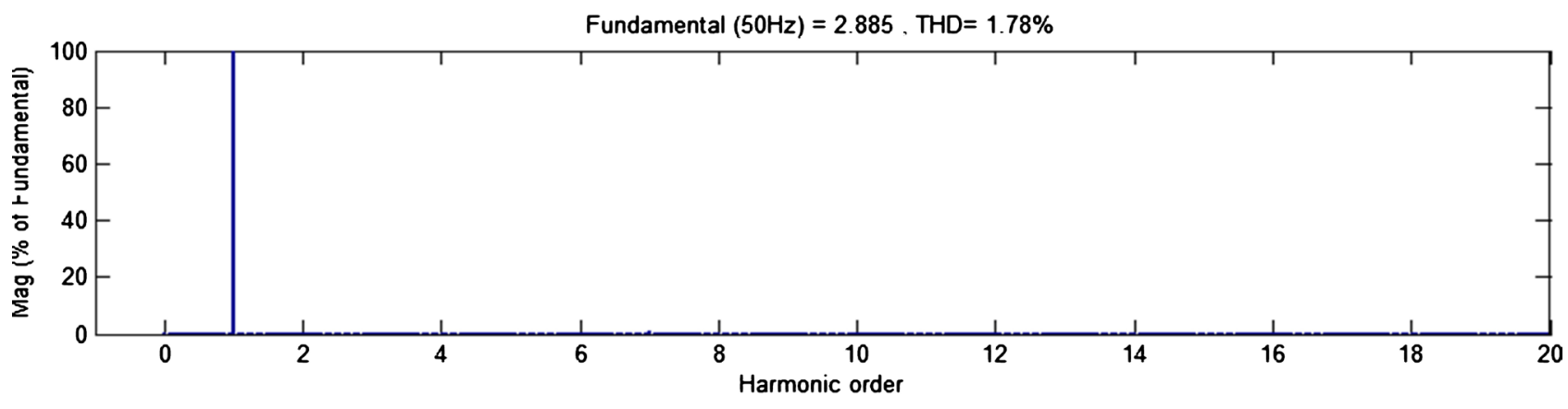

Figure 12. Current spectrum $i_{s a}$ after filtering for pulse width modulation (PWM) control. 


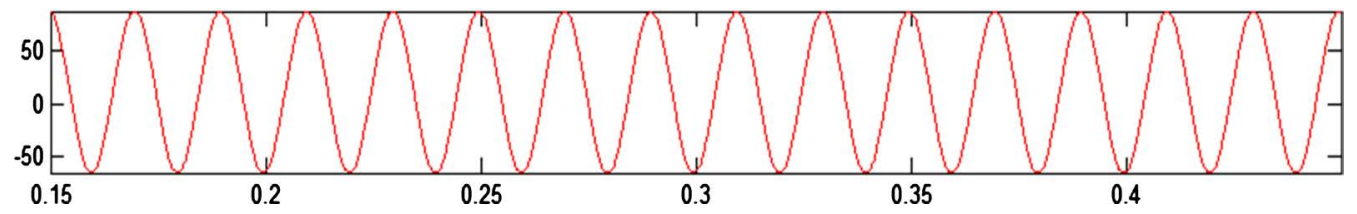

Figure 13. Current wave $i_{s a}$ after filtering for the Duty-Cycle Modulation command.

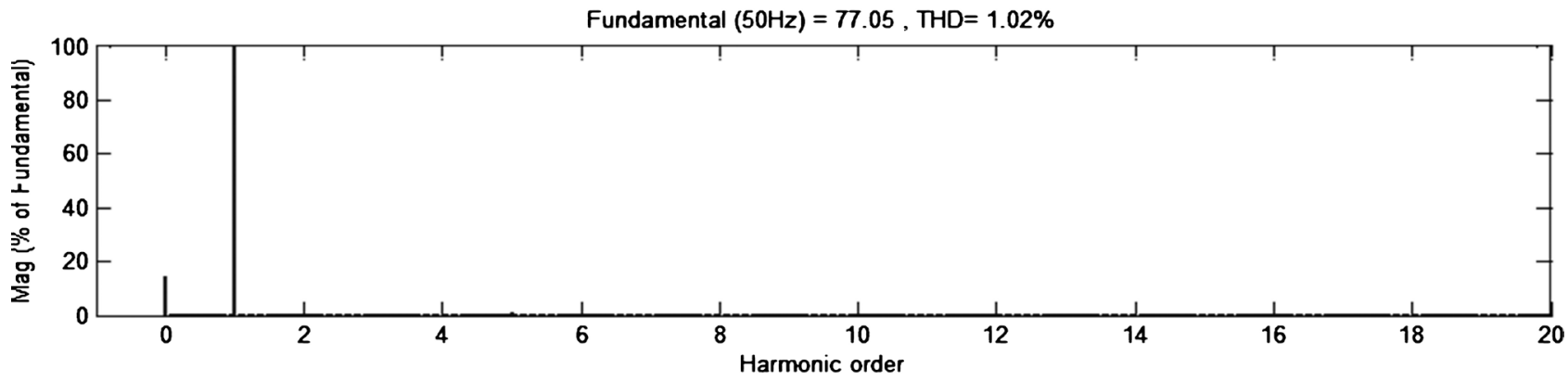

Figure 14. Current spectrum $i_{s a}$ after filtering for Duty-Cycle Modulation control.

519 standard and it can be seen that duty cycle control significantly improves the THD.

It is also observed in Figure 11 and Figure 13 that the current wave $i_{s a}$ for pulse-width control is noisier than that of Duty Cycle Modulation control, resulting in a significant improvement in the waveform.

Furthermore, the Duty Cycle Modulation drive is built around an operational amplifier (Figure 4), unlike the Pulse Width Modulation drive, which is built around two operational amplifiers, thus making the pulse width control drive more expensive.

\section{Conclusion}

In this paper, we have presented the operating principle of the active filter, given the simulink model of this filter and designed our active filter control strategy by Duty-Cycle Modulation capable of controlling the currents injected by this filter. The results obtained show a significant improvement on the harmonic current control techniques for the THD of $1.02 \%$ which was $26.25 \%$, the elimination of noise in the signal and the simplicity of the control with an easier implementation than the pulse width modulation make the Duty-Cycle Modulation control a very promising tool. The work in perspective is aimed at improving the inverter output filter to improve the control signal demodulation and spectral quality.

\section{Conflicts of Interest}

The authors declare no conflicts of interest regarding the publication of this paper.

\section{References}

[1] Schneider Electric. Guide de l'installation électrique-2009.

[2] Kanaan, H.Y. and Al-Haddad, K. (2004) Comparative Evaluation of Average Model Based Control Schemes Applied to a Three-Phase Series Active Power Filter for 
Voltage Harmonic Cancellation. Proceedings of $13^{\text {th }}$ IEEE International Symposium on Industrial Electronic (ISIE04), Ajaccio, France, 4-7 May 2004, 783-789. https://doi.org/10.1109/ISIE.2004.1571913

[3] Kanaan, H.Y., Hayek, A. and Al-Haddad, K. (2006) Nonlinear Control Design for a PWM Three-Phase Four-Wire Shunt Active Power Filter Based on the State-Space Averaged Model. Proceedings of 12 th International Conference on Harmonics and Quality of Power (ICHQP06), Cascais, Portugal, 1-5 October 2006.

[4] Montero, M.I.M., Cadaval, E.R. and Gonzalez, F.B. (2011) Comparison of Control Strategies for Shunt Active Power Filters in Three-Phase Four-Wire Systems. IEEE Transactions on Power Electronics, 22, 229-236.

https://doi.org/10.1109/TPEL.2006.886616

[5] Mbihi, J., Ndjali Beng, F. and Mbouenda, M. (2005) Modeling and Simulation of a Class of Duty-Cycle Modulators for Industrial Instrumentation. Iranian Journal of Electrical and Computer Engineering, 4, 121-128.

[6] Danwé Sounsoumou, Y.P., Djalo, H., Mbihi, J. and et Effa, J.Y. (2017) Modelisation Virtuelle d'un nouveau schema de réglage de Boost à commande rapprochée par modulation en rapport cyclique. Journal Afrique Science, 13, 176-185.

[7] Obono Biyobo, A., Nneme Nneme, L. and Mbihi, J. (2018) A Novel Sine Duty-Cycle Modulation Control Scheme for Photovoltaic Single-Phase Power Inverters. Wseas Transactions on Circuits and Systems, 17, 105-113.

[8] Akagi, H. (1998) Control Strategy and Site Selection of a Shunt Active Filter for Damping of Harmonic Propagation in Power Distribution Systems. Okayama University, Okayama. https://doi.org/10.1109/61.568259 